\title{
First-principles investigation of the very large perpendicular magnetic anisotropy at Fe|MgO and $\mathrm{Co} \mid \mathrm{MgO}$ interfaces
}

\author{
H. X. Yang, M. Chshiev, ${ }^{*}$ and B. Dieny \\ SPINTEC, UMR CEA/CNRS/UJF-Grenoble 1/Grenoble-INP, INAC, Grenoble, F-38054, France
}

J. H. Lee

SPINTEC, UMR CEA/CNRS/UJF-Grenoble 1/Grenoble-INP, INAC, Grenoble, F-38054, France, and Korea Institute of Science and Technology, Seoul 136-791, Korea

A. Manchon

SPINTEC, UMR CEA/CNRS/UJF-Grenoble 1/Grenoble-INP, INAC, Grenoble, F-38054, France, and King Abdullah University of Science and Technology, Thuwal 23955-6900, Saudi Arabia

\author{
K. H. Shin \\ Korea Institute of Science and Technology, Seoul 136-791, Korea \\ (Received 21 February 2011; revised manuscript received 22 April 2011; published 1 August 2011)
}

\begin{abstract}
The perpendicular magnetic anisotropy (PMA) arising at the interface between ferromagnetic transition metals and metallic oxides was investigated via first-principles calculations. In this work very large values of PMA, up to $3 \mathrm{erg} / \mathrm{cm}^{2}$, at $\mathrm{Fe} \mid \mathrm{MgO}$ interfaces are reported, in agreement with recent experiments. The origin of PMA is attributed to overlap between O- $p_{z}$ and transition metal $d_{z^{2}}$ orbitals hybridized with $d_{x z(y z)}$ orbitals with stronger spin-orbit coupling-induced splitting around the Fermi level for perpendicular magnetization orientation. Furthermore, it is shown that the PMA value weakens in the case of over- or underoxidation due to the fact that oxygen $p_{z}$ and transition metal $d_{z^{2}}$ orbital overlap is strongly affected by disorder, in agreement with experimental observations in magnetic tunnel junctions.
\end{abstract}

DOI: 10.1103/PhysRevB.84.054401

PACS number(s): 75.30.Gw, 72.25.-b, 73.40.Rw, 75.70.Cn

\section{INTRODUCTION}

Spin-orbit interaction (SOI) plays a major role in a wide class of physical phenomena from both fundamental and applications points of view. ${ }^{1}$ For instance, it is at the heart of basic magnetic phenomena such as magnetocrystalline anisotropy, ${ }^{2}$ the Rashba effect, ${ }^{3,4}$ and magnetization damping. Controlling SOI strength at the interface between ferromagnetic (FM) and nonmagnetic layers represents an outstanding challenge for advancement of transport and magnetic properties of spintronic magnetic devices, such as perpendicular magnetic tunnel junctions ${ }^{5-10}$ (p-MTJs) and tunneling anisotropic magnetoresistive (TAMR) systems. ${ }^{11,12}$ Recently, electric field control of interfacial magnetic anisotropy has attracted much attention as well. ${ }^{13,14}$ Traditionally, interfaces between magnetic and heavy nonmagnetic transition metals such as $\mathrm{Co}\left|\mathrm{Pt},{ }^{15} \mathrm{Co}\right| \mathrm{Pd},{ }^{16,17}$ and $\mathrm{Co} \mid \mathrm{Au}^{18}$ have been used to obtain perpendicular magnetic anisotropy (PMA). It has been shown that the onset of PMA at these interfaces is related to an increase in the orbital momentum of $\mathrm{Co}^{18}$ due to the strong hybridization between the $3 d$ orbitals of the transition metal and the $5 d$ orbitals of heavy metal. ${ }^{15}$ This hybridization enhances the energy splitting between the Co $3 d_{z^{2}}$ and the Co $3 d_{x^{2}-y^{2}}$ orbitals and induces a charge transfer between the two layers. ${ }^{19-21}$ As a result, the combination between SOI and hybridization-induced charge transfer leads to PMA. Thus, the presence of a heavy nonmagnetic layer ( $\mathrm{Pt}, \mathrm{Pd}, \mathrm{Au}, \mathrm{W}, \mathrm{Mo}$ ) was believed to be essential to obtain large PMA.

However, Monso et al. have shown that PMA could be observed also at $\mathrm{Co}(\mathrm{Fe}) \mid \mathrm{MOx}$ interfaces $(\mathrm{M}=\mathrm{Ta}, \mathrm{Mg}$,
$\mathrm{Al}, \mathrm{Ru}$, etc. $)^{22,23}$ despite the weak SOI at the interface. Surprisingly large PMA values, up to 1 to $2 \mathrm{erg} / \mathrm{cm}^{2}$, have been reported, which are comparable or even larger than the PMA observed at $\mathrm{Co} \mid \mathrm{Pt}$ or Co|Pd interfaces. ${ }^{24,25}$ This result is quite general and has been observed in both crystalline $(\mathrm{MgO})$ and amorphous (AlOx) barriers, using both natural or plasma oxidation. ${ }^{26,27}$ The PMA could be dramatically improved under annealing, ${ }^{10,28,29}$ and $\mathrm{x}$-ray photoemission spectroscopy has demonstrated that the PMA could be correlated without ambiguity with the presence of oxygen atoms at the interface. ${ }^{26,27}$ In fact, a correlation between PMA and oxidation conditions has been demonstrated for a wide range of FM|MOx including those based on $\mathrm{Co}_{x} \mathrm{Fe}_{1-x}$, thus indicating that the phenomenon is quite general at interfaces between magnetic transition metals and oxygen-terminated oxides. These observations led the authors to postulate that, despite the weak $\mathrm{SOI}$ of the elements $(\mathrm{Fe}, \mathrm{Co}, \mathrm{Al}, \mathrm{O})$, oxidation conditions play an essential role in PMA, as they do for TMR ${ }^{30}$ or interlayer exchange coupling. ${ }^{31}$ Recent experiments reported large PMA values of $1.3 \mathrm{erg} / \mathrm{cm}^{2}$ at $\mathrm{CoFeB} \mid \mathrm{MgO}$ structures. ${ }^{32,33}$ Furthermore, it has been demonstrated experimentally that there is a strong correlation between PMA and TMR maximum values obtained at the same optimal oxidation and annealing conditions. ${ }^{34}$

In this article, we report first-principles investigations of the PMA and the effect of interfacial oxidation conditions on the PMA at $\mathrm{Fe} \mid \mathrm{MgO}(100)$ structures. The latter can be viewed as a model system for $\mathrm{FM} \mid \mathrm{MOx}$ interfaces involving bcc electrodes including $\mathrm{Co}_{x} \mathrm{Fe}_{1-x}$ alloys. In agreement with experiments, it is demonstrated that despite the weak SOI, the 
bonding between the $\mathrm{Fe}-3 d$ and the $\mathrm{O}-2 p$ orbitals can give rise to PMA even stronger than that of $\mathrm{Co} \mid \mathrm{Pt}$ interfaces. The largest PMA value is obtained for ideal interfaces, while it is reduced for the case of over- or underoxidized interfaces. In addition, it is found that the FM|MgO PMA strength decreases when $\mathrm{Fe}$ is replaced by $\mathrm{Co}$, consistent with the recent report that PMA values decrease in $\mathrm{Co}_{x} \mathrm{Fe}_{1-x} \mid \mathrm{MgO}$ as the Co concentration increases. $^{35}$

\section{METHODOLOGY}

For $a b$ initio calculations, the Vienna ab initio simulation package (VASP) ${ }^{36}$ was used with the generalized gradient approximation $^{37}$ and projector augmented wave potentials. ${ }^{38}$ Calculations were performed in three steps. First, calculations were performed for both unrelaxed and relaxed structrures. In the case of relaxed structures, full structural relaxation in shape and volume was performed until the forces become lower than $0.001 \mathrm{eV} / \AA$ for determining the most stable interfacial geometries. Next, the Kohn-Sham equations were solved with no SOI taken into account to determine the charge distribution of the system ground state. Finally, the spin-orbit coupling was included and the total energy of the system was determined as a function of the orientation of the magnetic moments. A $19 \times 19 \times 3 K$-point mesh was used in our calculations, with the energy cutoff equal to $520 \mathrm{eV}$. Three structures were considered, as shown in Fig. 1(a) a "pure" (O-terminated) interface, (b) an overoxidized interface (with $\mathrm{O}$ inserted at the interfacial magnetic layer), and (c) an underoxidized (Mg-terminated) interface. The relaxed structures have been reported in our previous work. ${ }^{31}$ We point out that the situation of a "pure" interface is the most stable one, as observed in annealing experiments. ${ }^{28,29}$ The most stable location for the oxygen atoms is on top of metal ions due to the strong overlap between $\mathrm{Fe}-3 d$ and $\mathrm{O}-2 p$ orbitals. Correlatively, it is interesting to note that this structural configuration also yields the spin filtering phenomenon based on Bloch state
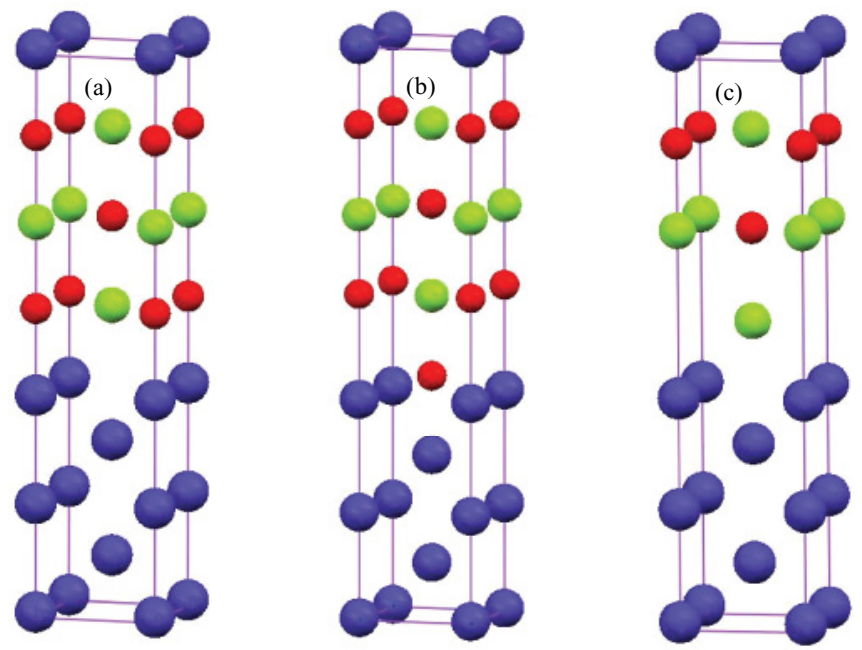

FIG. 1. (Color online) Schematics of the calculated crystalline structures for (a) pure, (b) overoxidized, and (c) underoxidized geometries. $\mathrm{Fe}, \mathrm{Mg}$, and $\mathrm{O}$ are represented by blue, green, and red balls, respectively.

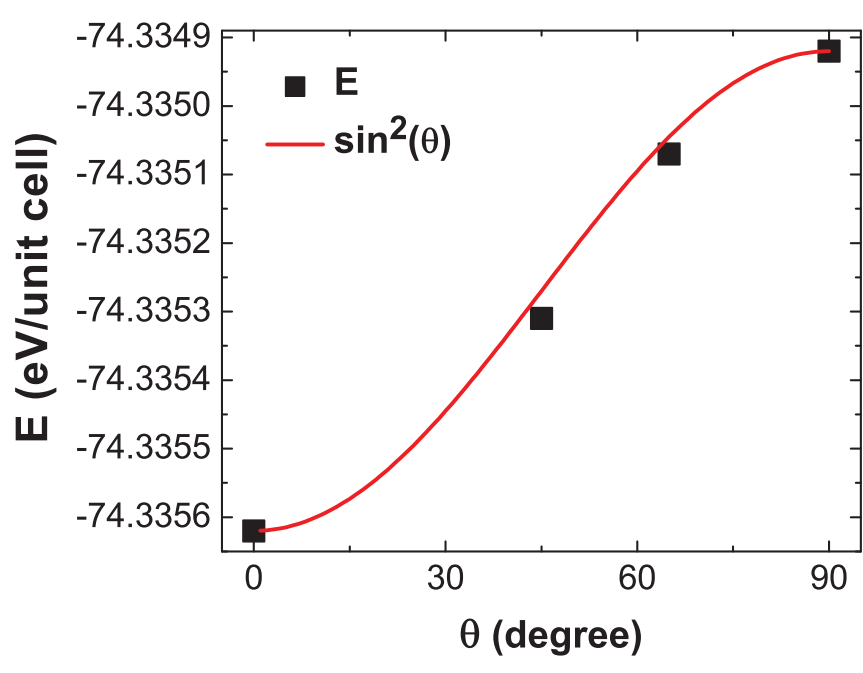

FIG. 2. (Color online) Angular dependence of the magnetic energy, where $\theta$ is the angle between the magnetization direction and the normal to the interface plane.

symmetry leading to high TMR values. ${ }^{39,40}$ Furthermore, the strong hybridization significantly modifies the band structure, giving rise to a high interfacial crystal field. ${ }^{19}$

\section{RESULTS AND DISCUSSION}

In Fig. 2, we present the calculated energy per unit cell as a function of the angle $\theta$ between magnetization orientation and normal to the plane for the pure unrelaxed $\mathrm{Fe} \mid \mathrm{MgO}$ interface. The dependence is well fitted by the conventional uniaxial anisotropy expression $E_{A}=K_{0}+K_{2} \sin ^{2} \theta$, where $K_{2}=0.7 \mathrm{meV} /$ atom $\left(K_{2}=1.36 \mathrm{erg} / \mathrm{cm}^{2}\right)$. Interestingly, the perpendicular surface anisotropy in this case is stronger than that of $\mathrm{Co} \mid \mathrm{Pt},{ }^{24,25}$ in agreement with recent experiments. ${ }^{32}$ The calculated anisotropy value is further enhanced for relaxed structures as reported in Table I, reaching the very large value of almost $3 \mathrm{erg} / \mathrm{cm}^{2}$ for the structure with three $\mathrm{MgO}$ monolayers shown in Fig. 1(a). It is interesting to note that the PMA is not strongly affected when the MgO thickness is increased up to 11 monolayers, giving the calculated value of $3.15 \mathrm{erg} / \mathrm{cm}^{2}$, which provides a good agreement with recent experiments. ${ }^{41}$ The PMA for relaxed $\mathrm{Fe} \mid \mathrm{MgO}$ structures weakens in the presence of interfacial disorder and becomes equal to 2.27 and $0.93 \mathrm{erg} / \mathrm{cm}^{2}$ for under- and overoxidized cases, respectively (see Table I), indicating that the oxidation

TABLE I. PMA value $\left(\mathrm{erg} / \mathrm{cm}^{2}\right)$ and magnetic moment $m\left[\mu_{B}\right.$ per $\mathrm{Fe}(\mathrm{Co})$ atom] for different layers of $\mathrm{Fe}(\mathrm{Co})$ in $\mathrm{Fe}(\mathrm{Co}) \mid \mathrm{MgO}$ magnetic tunnel junctions under different oxidation conditions.

\begin{tabular}{lcccc}
\hline \hline & \multicolumn{3}{c}{$\mathrm{Fe} \mid \mathrm{MgO}$} & $\mathrm{Co} \mid \mathrm{MgO}:$ \\
\cline { 2 - 4 } & Pure & Underoxidized & Overoxidized & pure \\
\hline PMA & 2.93 & 2.27 & 0.98 & 0.38 \\
$m\left(\mu_{B}\right)$ & & & 3.33 & 1.67 \\
Interfacial & 2.73 & 2.14 & 2.70 & 1.84 \\
Sublayer & 2.54 & 2.41 & 2.61 & 1.60 \\
Bulk & 2.56 & 2.55 & & \\
\hline \hline
\end{tabular}


conditions play a critical role in PMA as they do for both $\mathrm{TMR}^{30}$ and interlayer exchange coupling. ${ }^{31}$ Upon replacing Fe with Co, the PMA value decreases down to $0.38 \mathrm{erg} / \mathrm{cm}^{2}$, which agrees with report by Yakata et al. that Fe-rich $\mathrm{FeCoB}$ top free layers in $\mathrm{Co}_{60} \mathrm{Fe}_{20} \mathrm{~B}_{20}|\mathrm{MgO}|\left(\mathrm{Co}_{x} \mathrm{Fe}_{1-x}\right)_{80} \mathrm{~B}_{20}$ MTJs exhibit larger PMA than their Co-rich counterparts. ${ }^{35}$ Furthermore, the tendency for PMA to decrease with oxygen excess or deficit along the metal/oxide interface is consistent with the experimental observations of PMA dependence on annealing temperature and oxidation conditions. ${ }^{10,34}$ It was reported that at higher annealing temperatures, PMA increases due to interfacial quality improvement. ${ }^{10}$ Furthermore, upon varying the oxidation or annealing conditions, it was observed that the PMA reaches a maximum value for the same conditions under which the TMR ratio is also maximized, indicating that ideal interfaces are also crucial for PMA observation. ${ }^{34}$

In Table I we also report the evolution of interfacial Fe magnetic atomic moments as a function of distance from the interface. One can see that, compared to the pure case, the moments are enhanced or weakened for over- or underoxidized interfaces, respectively.

Let us now proceed with the explanation of the physical origin of the results obtained from first principles on the effect of oxidation conditions on PMA. To understand the PMA origin at $\mathrm{Fe} \mid \mathrm{MgO}$ interfaces, we performed a detailed analysis of the impact of SOI on electronic bands with out-of-plane $\left(d_{z^{2}}, d_{x z}, d_{y z}\right)$ and in-plane $\left(d_{x^{2}-y^{2}}, d_{x y}\right)$ Fe-3d and $\mathrm{O}-p_{z}$ orbital character.

We start from the analysis for pure interfaces represented in Fig. 1(a). In Fig. 3, we show bands around the Fermi

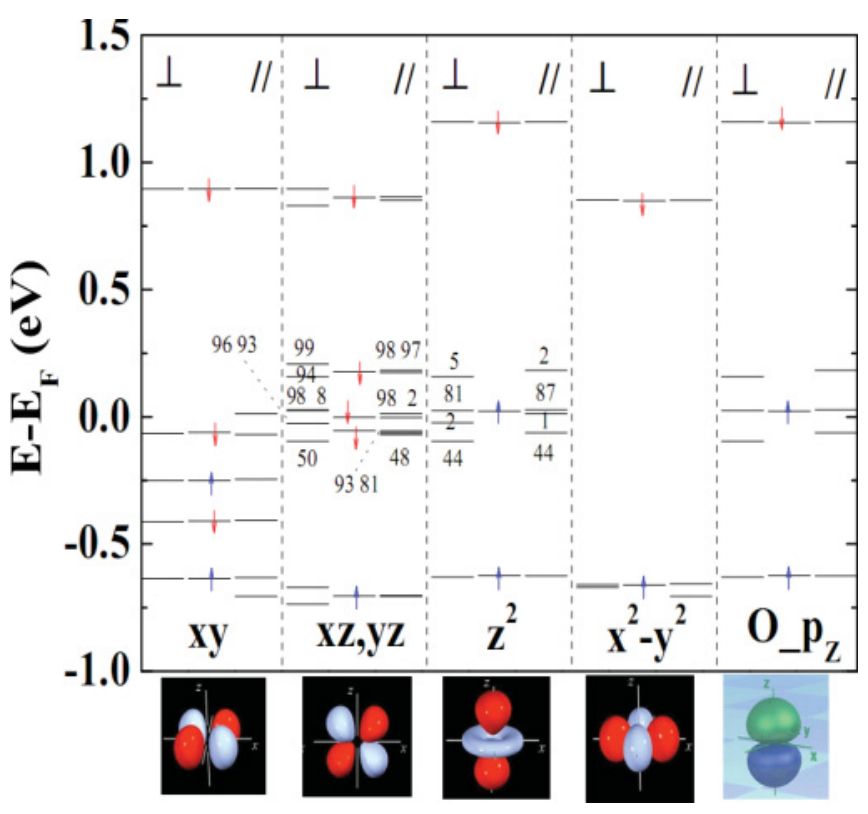

FIG. 3. (Color online) Spin-orbit coupling effects on wave function character at the $\bar{\Gamma}$ point of interfacial Fe $d$ and neighbor oxygen $p_{z}$ orbitals for the pure $\mathrm{Fe} \mid \mathrm{MgO}$ interface shown in Fig. 1(a). The three subcolumns in each column show the band levels for out-of-plane (left) and in-plane (right) orientations of magnetization as well as for the case with no spin-orbit interaction included (middle). Numbers are the percentage of orbital character components within Wigner-Seitz spheres around interfacial atoms. level $E_{F}$ at the $\bar{\Gamma}$ point with the orbital and interfacial atom projected wave function character for out-of-plane (left subcolumns) and in-plane (right subcolumns) orientation of the magnetization as well as in the absence of spin-orbit coupling (middle subcolumns). Let us concentrate on band levels in the immediate vicinity of the Fermi level. When no SOI is included (middle subcolumns), there are several doubledegenerated band levels with $d_{x z}$ and $d_{y z}$ character, which represent the minority Bloch states with $\Delta_{5}\left(p_{x}, p_{y}, d_{x z}, d_{y z}\right)$ symmetry. At the same time, there is a band level resulting from hybridization between $\mathrm{Fe}-d_{z^{2}}$ and $\mathrm{O}-p_{z}$ orbitals, which is a signature of the majority Bloch state with $\Delta_{1}\left(s, p_{z}, d_{z^{2}}\right)$ symmetry for $\mathrm{Fe}$ and $\mathrm{MgO}$ which is at the heart of the spin filtering phenomenon causing enhanced TMR values in MgO-based MTJs. ${ }^{39}$ When SOI is switched on, the picture is strongly modified. First, one can clearly see that the degeneracy is lifted for energy levels with a $d_{x z, y z}$ orbital character. Second, these levels become hybridized with Fe $d_{z^{2}}$, resulting in the appearance of additional levels of both $d_{z^{2}}$ and $d_{x z, y z}$ orbital character, represented by numbers showing the percentage of the corresponding orbital character components within Wigner-Seitz spheres (see Fig. 3). In particular, for the out-of-plane magnetization orientation (left subcolumns), the additional $d_{z^{2}}$ levels, with $5 \%, 2 \%$, and $44 \%$, originate from the $d_{x z, y z}$ orbital character due to SOI. For the same reason, the additional $d_{x z, y z}$ band, with $8 \%$, comes from the $d_{z^{2}}$ orbital. Furthermore, since the Fe- $d_{z^{2}}$ orbital has already been hybridized with $\mathrm{O}-p_{z}$ being a part of the $\Delta_{1}$ Bloch state, additional energy levels with $\mathrm{O}-p_{z}$ character also appear when SOI is switched on. This entire mechanism can be seen as spin-orbit-induced mixing between majority $\Delta_{1}$ and minority $\Delta_{5}$ Bloch states, which, by the way, may affect the amplitude of predicted high TMR ratios in MTJs. ${ }^{42}$ Finally, the hybridized band levels with $d_{z^{2}}, d_{x z}, d_{y z}$, and $p_{z}$ character are lower in energy, with a larger splitting for an out-of-plane magnetization orientation compared to the in-plane one, as clearly shown in the left and right subcolumns in Fig. 3, respectively. Thus, the lift of degeneracy of $d_{x z}$ and $d_{y z}$ orbitals, combined with the hybridizations between Fe- $d_{x z, y z}$ and $d_{z^{2}}$, as well as the hybridization between Fe- $d_{z^{2}}$ and $\mathrm{O}-p_{z}$ orbitals, is at the origin of PMA for pure $\mathrm{Fe} \mid \mathrm{MgO}$ interfaces. This result shows that the out-of-plane components of $d_{x z, y z}$ orbitals play a crucial role for PMA, similarly to $\mathrm{Co} \mid \mathrm{Pd}$ interfaces. $^{43}$

Next, we proceed with the same analysis for under- and overoxidized $\mathrm{Fe} \mid \mathrm{MgO}$ interfaces represented in Figs. 1(b) and 1(c), respectively. As shown in Fig. 4 for the case with an additional oxygen located at the Fe $\mid \mathrm{MgO}$ interface [Fig. 1(b)], spin orbit coupling again lifts the degeneracy for states with $d_{x z, y z}$, causing stronger splitting and a deeper level position for the out-of-plane orientation of magnetization compared to the in-plane one. However, the number of mixed states with both Fe- $d_{z^{2}}$ and O- $p_{z}$ orbitals is reduced due to the local charge redistribution induced by additional oxygen atoms. ${ }^{30}$ Since $d_{z^{2}}$ and $p_{z}$ orbital hybridization, which is one of the main causes of PMA, is not split, the anisotropy is significantly reduced.

A different picture occurs in the case of the underoxidized $\mathrm{Fe} \mid \mathrm{MgO}$ interface represented in Fig. 1(c). As shown in Fig. 5, the Fe- $d_{z^{2}}$ and $\mathrm{O}-p_{z}$ components around the Fermi level are now absent. As a result, the degeneracy lift induced by SOI 


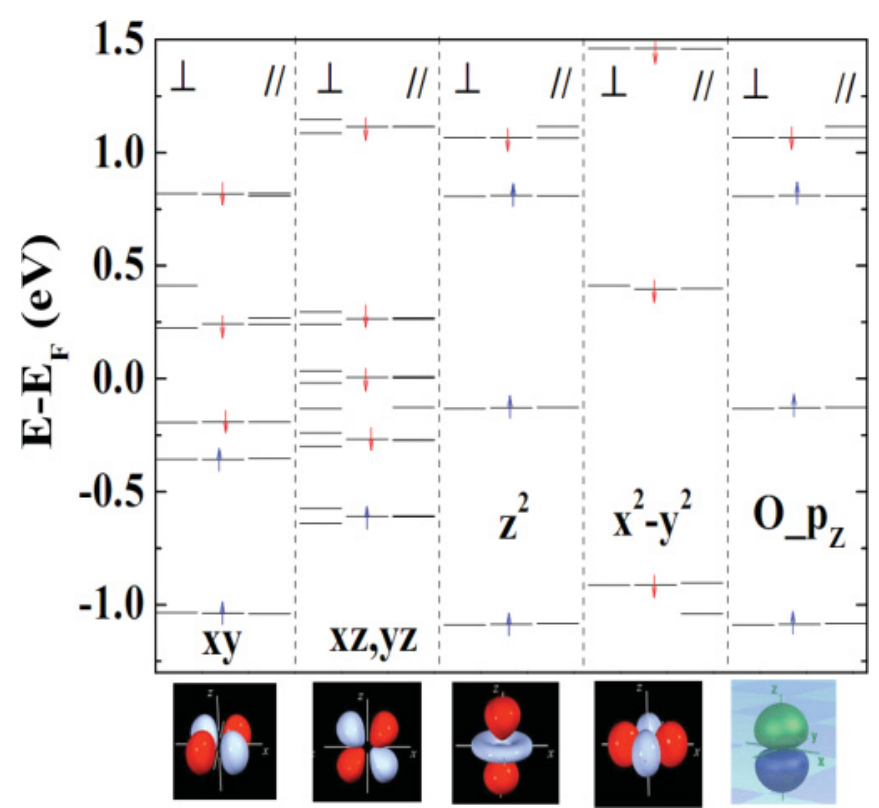

FIG. 4. (Color online) The same as Fig. 3, for an overoxidized $\mathrm{Fe} \mid \mathrm{MgO}$ interface.

for states with $d_{x z, y z}$ character is now solely responsible for the PMA. Since the splitting of these $d_{x z, y z}$ orbitals is still relatively strong and higher for the out-of-plane magnetization orientation compared to the in-plane one, anisotropy values are higher compared to the overoxidized case but lower compared to ideal $\mathrm{Fe} \mid \mathrm{MgO}$ interfaces. Thus, the PMA reaches its maximum for ideal interfaces. To understand the correlation between PMA and TMR, in Fig. 6 we have plotted the wave function character of the $\Delta_{1}$ Bloch state as a function of the position across the supercells used for PMA calculations. One can clearly see that the $\Delta_{1}$ decay rate is strongly enhanced in the case of the overoxidized

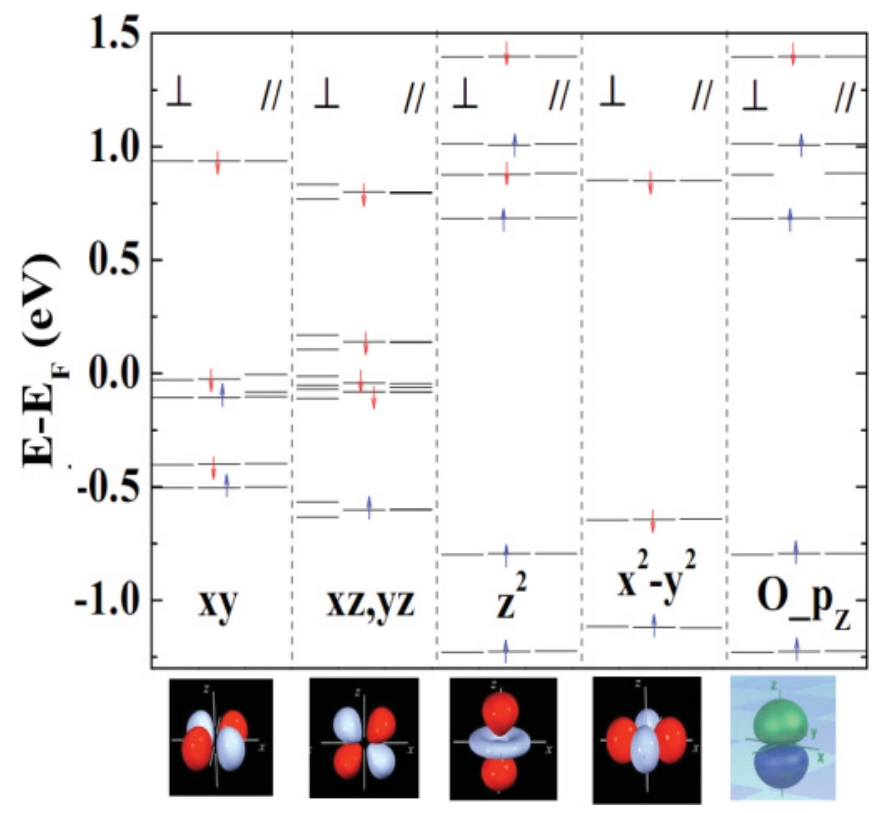

FIG. 5. (Color online) The same as Fig. 3, for an underoxidized $\mathrm{Fe} \mid \mathrm{MgO}$ interface.

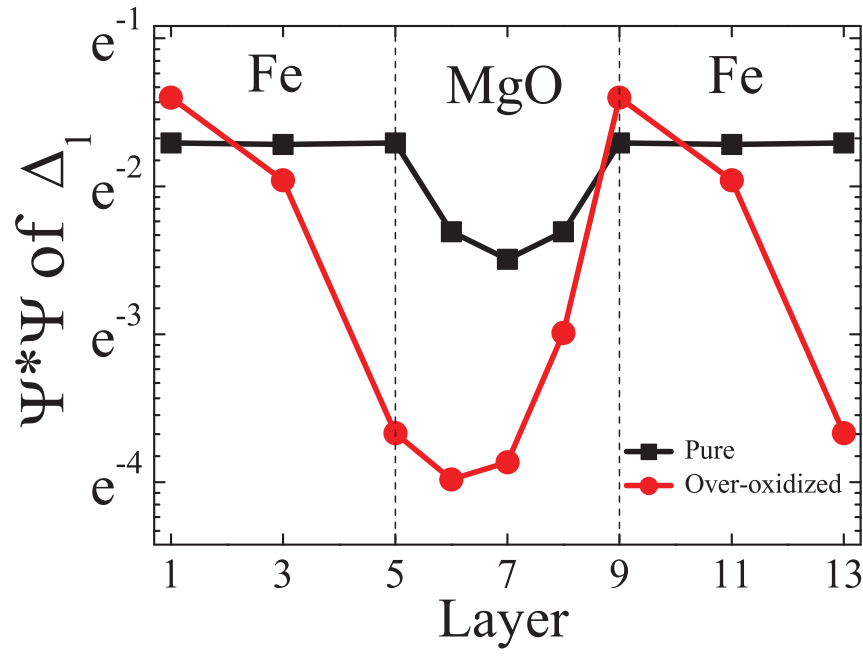

FIG. 6. (Color online) $\Delta_{1}$ Bloch state character at the $\Gamma$ point around the Fermi level as a function of layer number in the pure and overoxidized $\mathrm{Fe} \mid \mathrm{MgO}$ interfaces shown in Figs. 1(a) and 1(b), respectively. The $\Delta_{1}$ Bloch state is absent around the Fermi level in the underoxidized case shown in Fig. 1(c).

interface compared to the ideal one. ${ }^{44}$ There is no $\Delta_{1}$ band around the Fermi level for the underoxidized case as demonstrated above. This explains why both PMA and TMR reach their maximum values in a correlated way as observed experimentally, ${ }^{34}$ this maximum being reached for ideal interfaces.

\section{CONCLUSION}

In conclusion, we have presented $a b$ initio studies of PMA at $\mathrm{Fe} \mid \mathrm{MgO}$ interfaces as a function of the oxygen content along the interface. PMA values are higher in the case of pure interfaces, in agreement with recent experimental studies, ${ }^{32,34}$ and may reach up to $3 \mathrm{erg} / \mathrm{cm}^{2}$ for relaxed interfaces. The origin of the large PMA is ascribed to a combination of several factors: the degeneracy lift of out-of-plane $3 d$ orbitals, hybridizations between $\Delta_{1}\left(d_{z^{2}}\right)$ and $\Delta_{5}$-like $\left(d_{x z}\right.$ and $\left.d_{y z}\right) 3 d$ orbitals induced by SOI, and hybridizations between $\mathrm{Fe}-3 d$ and $\mathrm{O}-2 p$ orbitals at the interface between the transition metal and the insulator. The PMA amplitude decreases in the case of over- or underoxidized interfaces, in agreement with recent experiments.$^{10,34}$ This is due to the impact of splitting(disappearance) of $\Delta_{1}$-like hybridized states around the Fermi level in the presence(absence) of an additional oxygen atom. In addition, the PMA value is lower in the case of a $\mathrm{Co} \mid \mathrm{MgO}$ interface, which agrees with experimental findings that $\mathrm{Fe}$-rich $\mathrm{Co}_{x} \mathrm{Fe}_{1-x} \mathrm{~B} \mid \mathrm{MgO}$ structures have larger PMA than their Co-rich counterparts. ${ }^{35}$

\section{ACKNOWLEDGMENTS}

We thank L. Nistor, B. Rodmacq, A. Fert, H. Jaffres, O. Mryasov, A. Schuhl, and W. H. Butler for fruitful discussions. This work was supported by the Chair of Excellence Program of the Nanosciences Foundation in Grenoble, France, the ERC Advanced Grant Hymagine, and the KRCF DRC program. 
*mair.chshiev@cea.fr

${ }^{1}$ J. Fabian, A. Matos-Abiague, C. Ertler, P. Stano, and I. Zutic, Acta Phys. Slov. 57, 565 (2007).

${ }^{2}$ A. Matos-Abiague and J. Fabian, Phys. Rev. B 79, 155303 (2009).

${ }^{3}$ Yu A. Bychkov and E. I. Rashba, J. Phys. C 17, 6039 (1984).

${ }^{4}$ I. Zutic, J. Fabian, and S. Das Sarma, Rev. Mod. Phys. 76, 323 (2004).

${ }^{5}$ K. Mizunuma, S. Ikeda, J. H. Park, H. Yamamoto, H. Gan, K. Miura, H. Hasegawa, J. Hayakawa, F. Matsukura, and H. Ohno, Appl. Phys. Lett. 95, 232516 (2009).

${ }^{6}$ G. Kim, Y. Sakuraba, M. Oogane, Y. Ando, and T. Miyazaki, Appl. Phys. Lett. 92, 172502 (2008).

${ }^{7}$ C. Ducruet, B. Carvello, B. Rodmacq, S. Auffret, G. Gaudin, and B. Dieny, J. Appl. Phys. 103, 07A918 (2008).

${ }^{8}$ J.-H. Park, C. Park, T. Jeong, M. T. Moneck, N. T. Nufer, and J.-G. Zhu, J. Appl. Phys. 103, 07A917 (2008).

${ }^{9}$ D. Lim, S. Kim, and S. R. Lee, J. Appl. Phys. 97, 10 C902 (2005).

${ }^{10}$ L. E. Nistor, B. Rodmacq, S. Auffret, and B. Dieny, Appl. Phys. Lett. 94, 012512 (2009).

${ }^{11}$ L. Gao, X. Jiang, S.-H. Yang, J. D. Burton, E. Y. Tsymbal, and Stuart S. P. Parkin, Phys. Rev. Lett. 99, 226602 (2007).

${ }^{12}$ B. G. Park, J. Wunderlich, D. A. Williams, S. J. Joo, K. Y. Jung, K. H. Shin, K. Olejnik, A. B. Shick, and T. Jungwirth, Phys. Rev. Lett. 100, 087204 (2008).

${ }^{13}$ Y. Shita, T. Maruyama, T. Nozaki, T. Shinjo, M. Shiraishi, and Y. Suzuki, Appl. Phys. Express 2, 063001 (2009).

${ }^{14}$ K. Nakamura, T. Akiyama, T. Ito, M. Weinert, and A. J. Freeman, Phys. Rev. B 81, 220409(R) (2010).

${ }^{15}$ N. Nakajima, T. Koide, T. Shidara, H. Miyauchi, H. Fukutani, A. Fujimori, K. Iio, T. Katayama, M. Nyvlt, and Y. Suzuki, Phys. Rev. Lett. 81, 5229 (1998).

${ }^{16}$ P. F. Carcia, A. D. Meinhaldt, and A. Suna, Appl. Phys. Lett. 47, 178 (1985).

${ }^{17}$ H. J. G. Draaisma, W. J. M. de Jonge, and F. J. A. den Broeder, J. Magn. Magn. Mater. 66, 351 (1987).

${ }^{18}$ D. Weller, Y. Wu, J. Stohr, M. G. Samant, B. D. Hermsmeier, and C. Chappert, Phys. Rev. B 49, 12888 (1994).

${ }^{19}$ P. Bruno, Phys. Rev. B 39, 865 (1989).

${ }^{20}$ G. H. O. Daalderop, P. J. Kelly, and M. F. H. Schuurmans, Phys. Rev. B 50, 9989 (1994).

${ }^{21}$ K. Kyuno, R. Yamamoto, and S. Asano, J. Phys. Soc. Jpn. 61, 2099 (1992).

${ }^{22}$ S. Monso, B. Rodmacq, S. Auffret, G. Casali, F. Fettar, B. Gilles, B. Dieny, and P. Boyer, Appl. Phys. Lett. 80, 4157 (2002).

${ }^{23}$ B. Rodmacq, S. Auffret, B. Dieny, S. Monso, and P. Boyer, J. Appl. Phys. 93, 7513 (2003).
${ }^{24}$ V. W. Guo, B. Lu, X. Wu, G. Ju, B. Valcu, and D. Weller, J. Appl. Phys. 99, 08E918 (2006).

${ }^{25}$ M. T. Johnson, R. Jungblut, P. J. Kelly, and F. J. A. den Broeder, J. Magn. Magn. Mater. 148, 118 (1995).

${ }^{26}$ D. Lacour, M. Hehn, M. Alnot, F. Montaigne, F. Greullet, G. Lengaigne, O. Lenoble, S. Robert, and A. Schuhl, Appl. Phys. Lett. 90, 192506 (2007).

${ }^{27}$ A. Manchon, C. Ducruet, L. Lombard, S. Auffret, B. Rodmacq, B. Dieny, S. Pizzini, J. Vogel, V. Uhlír, M. Hochstrasser, and G. Panaccione, J. Appl. Phys. 104, 043914 (2008).

${ }^{28}$ A. Manchon, S. Pizzini, J. Vogel, V. Uhlír, L. Lombard, C. Ducruet, S. Auffret, B. Rodmacq, B. Dieny, M. Hochstrasser, and G. Panaccione, J. Magn. Magn. Mater. 320, 1889 (2008).

${ }^{29}$ B. Rodmacq, A. Manchon, C. Ducruet, S. Auffret, and B. Dieny, Phys. Rev. B 79, 024423 (2009).

${ }^{30}$ X.-G. Zhang, W. H. Butler, and A. Bandyopadhyay, Phys. Rev. B 68, 092402 (2003).

${ }^{31}$ H. X. Yang, M. Chshiev, A. Kalitsov, A. Schuhl, and W. H. Butler, Appl. Phys. Lett. 96, 262509 (2010).

${ }^{32}$ S. Ikeda, K. Miura, H. Yamamoto, K. Mizunuma, H. D. Gan, M. Endo, S. Kanai, J. Hayakawa, F. Matsukura, and H. Ohno, Nat. Mater. 9, 721 (2010).

${ }^{33}$ M. Endo, S. Kanai, S. Ikeda, F. Matsukura, and H. Ohno, Appl. Phys. Lett. 96, 212503 (2010).

${ }^{34}$ L. E. Nistor, B. Rodmacq, C. Ducruet, C. Portemont, I. L. Prejbeanu, and B. Dieny, Magnetics, IEEE Trans. Magn. 46, 1412 (2010).

${ }^{35}$ S. Yakata, H. Kubota, Y. Suzuki, K. Yakushiji, A. Fukushima, S. Yuasa, and K. Ando, J. Appl. Phys. 105, 07D131 (2009).

${ }^{36}$ G. Kresse and J. Hafner, Phys. Rev. B 47, 558 (1993); G. Kresse and J. Furthmuller, ibid. 54, 11169 (1996); Comput. Mater. Sci. 6, 15 (1996).

${ }^{37}$ Y. Wang and J. P. Perdew, Phys. Rev. B 44, 13298 (1991).

${ }^{38}$ P. E. Blöchl, Phys. Rev. B 50, 17953 (1994); G. Kresse and D. Joubert, ibid. 59, 1758 (1999).

${ }^{39}$ W. H. Butler, X.-G. Zhang, T. C. Schulthess, and J. M. MacLaren, Phys. Rev. B 63, 054416 (2001).

${ }^{40}$ J. Mathon and A. Umerski, Phys. Rev. B 63, 220403(R) (2001).

${ }^{41}$ M. Yamanouchi, R. Koizumi, S. Ikeda, H. Sato, K. Mizunuma, K. Miura, H. D. Gan, F. Matsukura, and H. Ohno, J. Appl. Phys. 109, 07 C712 (2011)

${ }^{42}$ A. Vedyayev, N. Ryzhanova, N. Strelkov, M. Chshiev, and B. Dieny, J. Appl. Phys. 107, 09C720 (2010).

${ }^{43}$ D. S. Wang, R. Wu, and A. J. Freeman, Phys. Rev. B 48, 15886 (1993).

${ }^{44}$ W. H. Butler, X.-G. Zhang, S. Vutukuri, M. Chshiev, and T. C. Schulthess, IEEE Trans. Magn. 41, 2645 (2005). 